\title{
CHERN NUMBERS OF THE MODULI SPACE OF SPATIAL POLYGONS
}

\author{
YASUHIKO KAMIYAMA
}

\begin{abstract}
Let $\mathscr{M}_{n}(n \geq 3)$ be the moduli space of spatial polygons with $n$ edges. We consider the case of odd $n$. First we establish a procedure to determine the Chern numbers of $\mathscr{M}_{n}$. Next we follow the procedure and get a description of $\mathscr{M}_{n}(n \leq 9)$ in the complex cobordism group $\Omega_{2 n-6}^{U}$. Finally we determine some characteristic numbers of $\mathscr{M}_{n}$. In particular, we calculate the Todd genus of $\mathscr{M}_{n}$ by showing that $\mathscr{M}_{n}$ is birationally equivalent to $C P^{n-3}$.
\end{abstract}

\section{Introduction}

Let $\mathscr{M}_{n}(n \geq 3)$ be the moduli space of spatial polygons $P=\left(a_{1}, a_{2}, \ldots, a_{n}\right)$ whose edges are vectors $a_{i} \in \boldsymbol{R}^{3}$ of length $\left|a_{i}\right|=1 \quad(1 \leq i \leq n)$. Two polygons are identified if they differ only by motions in $R^{3}$. The sum of the vectors is assumed to be zero. Thus:

$$
\mathscr{M}_{n}=\left\{P=\left(a_{1}, \ldots, a_{n}\right) \in\left(S^{2}\right)^{n}: a_{1}+\cdots+a_{n}=0\right\} / S O(3) .
$$

It is known that $\mathscr{M}_{n}$ is a Kähler manifold of complex dimension $n-3$. For odd $n$ or $n=4, \mathscr{M}_{n}$ has no singular points. For even $n$ with $n \geq 6, P=$ $\left(a_{1}, a_{2}, \ldots, a_{n}\right)$ is a singular point if and only if all the $a_{i}(1 \leq i \leq n)$ lie on a line in $\boldsymbol{R}^{3}$ through $O$. Such singular points are cone-like singularities and have neighborhoods $C\left(S^{n-3} \times S_{S^{1}} S^{n-3}\right)$, where $C$ denotes the cone and $S^{1}$ acts on both copies of $S^{n-3}$ by complex multiplication (see for example [8]).

For odd $n$, the module $H_{*}\left(\mathscr{M}_{n} ; \boldsymbol{R}\right)$ was determined by Kirwan and Klyachko $[10],[12]$. Later the cohomology ring $H^{*}\left(\mathscr{M}_{n} ; \boldsymbol{R}\right)$ was determined by Brion and Kirwan [1], [11] (cf. Theorem 2.2). In particular $H^{*}\left(\mathscr{M}_{n} ; \boldsymbol{R}\right)$ is generated by certain two dimensional cohomology classes.

In contrast to this, for even $n, H_{*}\left(\mathscr{M}_{n} ; \boldsymbol{R}\right)$ is complicated and is not generated by two dimensional cohomology classes nor does not obey Poincaré duality [5]. The cohomology ring $H^{*}\left(\mathscr{M}_{n} ; \boldsymbol{R}\right)$ is not yet known.

1991 Mathematics Subject Classification: 57R20, 57R77

Key words and phrases: spatial polygon, Chern number, complex cobordism.

Received September 3, 1999. 
For the rest of this paper, we assume $n$ to be odd and set $n=2 m+1$. In [6], we described $\mathscr{M}_{n}$ in the oriented cobordism group $\Omega_{2 n-6}^{S O}$. The result is that $\mathscr{M}_{n}$ is oriented cobordant to $(-1)^{m+1}\left(\begin{array}{c}2 m-1 \\ m\end{array}\right) C P^{2 m-2}$. Here $\left(\begin{array}{c}2 m-1 \\ m\end{array}\right)$ denotes the binomial coefficient, and we give an orientation to $\mathscr{M}_{n}$ which is induced from the complex structure. The proof of this fact is carried out by constructing an oriented manifold with boundary, which gives the desired cobordism. However, such a method seems difficult when we describe $\mathscr{M}_{n}$ in the complex cobordism group $\Omega_{2 n-6}^{U}$, where $2 n-6$ denotes the real dimension.

The main topic of this paper is a description of $\mathscr{M}_{n}$ in $\Omega_{2 n-6}^{U}$. Since $\mathscr{M}_{3}=$ \{point\}, the problem is trivial for $\mathscr{M}_{3}$. Hence for the rest of this paper, we assume $n$ to be odd $\geq 5$. Recall that $\Omega_{*}^{U}$ is determined by the Chern numbers [14]. Hence the problem is essentially to determine the Chern numbers of $\mathscr{M}_{n}$. In fact, we have a procedure to determine such numbers:

TheOREM A. We have a procedure to determine the Chern numbers of $\mathscr{M}_{n}$.

For more details of Theorem A, see Section 2. The key theorems for Theorem A are as follows.

(i) First we give the ring structure of $H^{*}\left(\mathscr{M}_{n} ; \boldsymbol{R}\right)$ in Theorem 2.2. In particular, $H^{*}\left(\mathscr{M}_{n} ; \boldsymbol{R}\right)$ is generated by certain two dimensional cohomology classes $z_{1}, \ldots, z_{n} \in H^{2}\left(\mathscr{M}_{n} ; \boldsymbol{R}\right)$.

(ii) Next for a sequence $\left(d_{1}, \ldots, d_{n}\right)$ of nonnegative integers with $\sum_{l=1}^{n} d_{i}=$ $n-3$, we give the intersection number $\left\langle z_{1}^{d_{1}} \cdots z_{n}^{d_{n}}, \mu_{\mathscr{M}_{n}}\right\rangle$ in Theorems 2.5 and 2.6, where $\mu_{\mathscr{M}_{n}}$ denotes the fundamental homology class determined by the orientation which is induced from the complex structure on $\mathscr{M}_{n}$.

(iii) Finally we describe $c\left(\mathscr{M}_{n}\right)$, the total Chern class of the tangent bundle of $\mathscr{M}_{n}$, in terms of $z_{1}, \ldots, z_{n} \in H^{2}\left(\mathscr{M}_{n} ; \boldsymbol{R}\right)$ (cf. (i)) in Theorem 2.8.

From (iii), we can describe $c_{i}\left(\mathscr{M}_{n}\right)$ in terms of $z_{1}, \ldots, z_{n}$ (cf. Theorems 2.8 and 2.12). Then for each partition $I=i_{1}, \ldots, i_{r}$ of $n-3$, the $I$-th Chern number $c_{I}\left[\mathscr{M}_{n}\right]=c_{l_{1}, \ldots, l_{r}}\left[\mathscr{M}_{n}\right]$ is determined from (ii). (As usual, we set $c_{i_{1}, \ldots, l_{r}}\left[\mathscr{M}_{n}\right]=$ $\left\langle c_{l_{1}}\left(\mathscr{M}_{n}\right) \cdots c_{l_{r}}\left(\mathscr{M}_{n}\right), \mu_{\mathscr{M}_{n}}\right\rangle$.)

Theorem $\mathrm{A}$ is effective for the calculations of the Chern numbers of $\mathscr{M}_{n}$. In fact, we give the results for $n \leq 9$ in Theorem 3.1. Using these results, we have the following:

THEOREM B. (i) In $\Omega_{4}^{U}$, we have

$$
\left[\mathscr{M}_{5}\right]=4\left[C P^{1} \times C P^{1}\right]-3\left[C P^{2}\right] .
$$

(ii) In $\Omega_{8}^{U}$, we have

$$
\begin{aligned}
{\left[\mathscr{M}_{7}\right]=} & -9\left[\left(C P^{1}\right)^{4}\right]+33\left[\left(C P^{1}\right)^{2} \times C P^{2}\right]-33\left[C P^{1} \times C P^{3}\right] \\
& +0\left[\left(C P^{2}\right)^{2}\right]+10\left[C P^{4}\right] .
\end{aligned}
$$


(iii) In $\Omega_{12}^{U} \otimes Q$, we have

$$
\begin{aligned}
{\left[\mathscr{M}_{9}\right]=} & 43\left[\left(\boldsymbol{C} P^{1}\right)^{6}\right]-\frac{668}{3}\left[\left(\boldsymbol{C} P^{1}\right)^{4} \times C P^{2}\right]+234\left[\left(\boldsymbol{C} P^{1}\right)^{3} \times C P^{3}\right] \\
& +220\left[\left(C P^{1}\right)^{2} \times\left(C P^{2}\right)^{2}\right]-220\left[\left(C P^{1}\right)^{2} \times C P^{4}\right]+\frac{440}{3}\left[C P^{1} \times C P^{5}\right] \\
& -220\left[C P^{1} \times C P^{2} \times C P^{3}\right]+0\left[\left(C P^{2}\right)^{3}\right]+0\left[C P^{2} \times C P^{4}\right] \\
& +55\left[\left(C P^{3}\right)^{2}\right]-35\left[C P^{6}\right] .
\end{aligned}
$$

Remark 1.2. The rational coefficients $-668 / 3$ and $440 / 3$ in Theorem B (iii) are due to the fact that $\left[C P^{5}\right] \in \Omega_{10}^{U}$ is not a ring generator of $\Omega_{*}^{U}[14]$. Instead of $\left[\boldsymbol{C} P^{5}\right]$, if we use $x_{5}$ defined by $x_{5}=\left[\boldsymbol{C} P^{5}\right]+\left[H_{3,3}\right]-\left[H_{2,4}\right]$, then we obtain a description of $\left[\mathscr{M}_{9}\right]$ in $\Omega_{12}^{U}$. For more details, see Remark 3.2.

Finally we consider the case of general odd $n$. Note that for each partition $I=i_{1}, \ldots, i_{r}$ of $n-3$, the $I$-th Chern number $c_{I}\left[\mathscr{M}_{n}\right]$ is defined. In this paper, instead of giving all the $c_{I}\left[\mathscr{M}_{n}\right]$, we give some characteristic numbers of $\mathscr{M}_{n}$.

Recall that for a compact, complex $k$-dimensional manifold $M$, the Todd genus $T[M]$ and a certain, well-known integral combination of the Chern numbers $s_{k}[M]$ are defined as follows (see for example [13]). First let $\left\{T_{k}\right\}$ be the multiplicative sequence of polynomials belonging to the power series $f(x)=$ $x /\left(1-e^{-x}\right)$. Then the Todd genus $T[M]$ is defined to be the characteristic number $\left\langle T_{k}\left(c_{1}(M), \ldots, c_{k}(M)\right), \mu_{M}\right\rangle$.

Next let $\sigma_{l}$ denote the $i$-th elementary symmetric polynomial in variables $t_{1}, \ldots, t_{k}$, and let $s_{k}\left(\sigma_{1}, \ldots, \sigma_{k}\right)$ denote the polynomial in $\sigma_{l}$ which express the sum $t_{1}^{k}+\cdots+t_{k}^{k}$. Then $s_{k}[M]$ is defined to be the characteristic number $\left\langle s_{k}\left(c_{1}(M), \ldots, c_{k}(M)\right), \mu_{M}\right\rangle$. (The characteristic number $s_{k}[M]$ is important. For example if $s_{k}[M] \neq 0$, then $M$ cannot be expressed non-trivially as a product of complex manifolds.)

Then we have the following results on characteristic numbers of $\mathscr{M}_{n}$. As before, we set $n=2 m+1$.

THEOREM C. (i) We have

$$
c_{1}^{n-3}\left[\mathscr{M}_{n}\right]=\sum_{j=0}^{m-1}(-1)^{J}\left(\begin{array}{c}
2 m \\
j
\end{array}\right)(2 m-1-2 j)^{2 m-2} .
$$

(ii) We have

$$
c_{n-3}\left[\mathscr{M}_{n}\right]=-2^{2 m-1}+(2 m+1)\left(\begin{array}{c}
2 m-1 \\
m
\end{array}\right) .
$$

(iii) We have

$$
T\left[\mathscr{M}_{n}\right]=1 .
$$


(iv) We have

$$
s_{n-3}\left[\mathscr{M}_{n}\right]=(-1)^{m+1}(2 m-1)\left(\begin{array}{c}
2 m-1 \\
m
\end{array}\right)
$$

In fact we can deduce Theorem $\mathrm{C}$ (iii) from the following stronger assertion, which may be of interest in their own right (cf. Assertion 4.1): $\mathscr{M}_{n}$ is birationally equivalent to $C P^{n-3}$. And as examples for other Chern numbers, we give $c_{1} c_{n-4}\left[\mathscr{M}_{n}\right]$ and $c_{2} c_{n-5}\left[\mathscr{M}_{n}\right]$ in Theorem 4.5.

Remark 1.3. (i) It is known that $\mathscr{M}_{n}$ admits a symplectic structure [8], [12]. Let $\omega_{n}$ be the symplectic form on $\mathscr{M}_{n}$. Then it is known that $\left[\omega_{n}\right]=c_{1}\left(\mathscr{M}_{n}\right) \in$ $H^{2}\left(\mathscr{M}_{n} ; \boldsymbol{R}\right)$ [2] (cf. Remark 2.13). Thus Theorem C (i) gives the symplectic volume $\left\langle\omega_{n}^{n-3}, \mu_{\mathscr{M}_{n}}\right\rangle$ of $\mathscr{M}_{n}$.

(ii) From the fact that $\mathscr{M}_{n}$ is oriented cobordant to $(-1)^{m+1}\left(\begin{array}{c}2 m-1 \\ m\end{array}\right)$. $C P^{2 m-2}[6]$, we can determine all the Pontrjagin numbers and the Stiefel-Whitney numbers of $\mathscr{M}_{n}$.

This paper is organized as follows. In Section 2, we study Theorem A in detail. We explain how to compute the Chern numbers of $\mathscr{M}_{n}$ according to the steps (i), (ii) and (iii) in this section. In Section 3 we prove Theorem B, and in Section 4 we prove Theorem $C$.

\section{Procedure for the Chern numbers of $\mathscr{M}_{n}$}

In this section, we study Theorem $\mathrm{A}$ in detail. First we recall the structure of $H^{*}\left(\mathscr{M}_{n} ; \boldsymbol{R}\right)$ for odd $n$, which was determined by Brion and Kirwan [1], [11]. For $i \in\{1, \ldots, n\}$, we define $A_{n, l} \subset\left(\boldsymbol{R}^{3}\right)^{n}$ by

$$
A_{n, l}=\left\{P=\left(a_{1}, \ldots, a_{n}\right) \in\left(S^{2}\right)^{n}: a_{1}+\cdots+a_{n}=0 \text { and } a_{i}=\left(\begin{array}{l}
0 \\
0 \\
1
\end{array}\right)\right\} \text {. }
$$

Let $S O(2)$ act on $\boldsymbol{R}^{3}$ by rotation about the $z$-axis. Then for odd $n$, the diagonal $S O(2)$-action on $\left(\boldsymbol{R}^{3}\right)^{n}$ is free on $A_{n, l}$ and we have $\mathscr{M}_{n}=A_{n, l} / S O(2)$ (cf. (1.1)). Therefore, $A_{n, l} \rightarrow \mathscr{M}_{n}$ is a principal $S O(2)$-bundle. Let $\xi_{l} \rightarrow \mathscr{M}_{n}$ be a complex line bundle associated with $A_{n, l} \rightarrow \mathscr{M}_{n}$ :

$$
\xi_{l}=\left(A_{n, l} \times C\right) / S^{1}
$$

where we identify $S O(2)$ with $S^{1}$ and let $S^{1}$ act on $A_{n, l} \times C$ by

$$
(P, \alpha) \cdot g=(P g, \alpha g), \quad(P, \alpha) \in A_{n, l} \times C, \quad g \in S^{1} .
$$

Then we define $z_{l} \in H^{2}\left(\mathscr{M}_{n} ; \boldsymbol{R}\right)$ to be the Chern class of $\xi_{l}$ :

$$
z_{l}=c_{1}\left(\xi_{l}\right), \quad 1 \leq i \leq n .
$$

Now we have the following theorem. 
THEOREM $2.2[1],[11]$. When $n=2 m+1$, the algebra $H^{*}\left(\mathscr{M}_{n} ; \boldsymbol{R}\right)$ is generated by $z_{1}, \ldots, z_{n}$ with the relations:

(i) $z_{1}^{2}=\cdots=z_{n}^{2}$.

(ii) $\prod_{J \in J}\left(z_{l}+z_{J}\right)=0$, for all $1 \leq i \leq n$ and $J \subset\{1, \ldots, n\}$ such that $i \notin J$ and card $(J)=m$, where card denotes the cardinal.

Next we study the intersection numbers. For a sequence $\left(d_{1}, \ldots, d_{n}\right)$ of nonnegative integers with $\sum_{l=1}^{n} d_{i}=n-3$, we define $\left\langle\tau_{d_{1}} \cdots \tau_{d_{n}}\right\rangle$ by

$$
\left\langle\tau_{d_{1}} \cdots \tau_{d_{n}}\right\rangle=\left\langle z_{1}^{d_{1}} \cdots z_{n}^{d_{n}}, \mu_{\mathscr{M}_{n}}\right\rangle
$$

where $z_{l} \in H^{2}\left(\mathscr{M}_{n} ; \boldsymbol{R}\right)(1 \leq i \leq n)$ is defined in $(2.1)$, and $\mu_{\mathscr{M}_{n}}$ denotes the fundamental homology class of $\mathscr{M}_{n}$. Thus we need to determine $\left\langle\tau_{d_{1}} \cdots \tau_{d_{n}}\right\rangle$ for all $\left(d_{1}, \ldots, d_{n}\right)$. To do this, we consider the following types of $\left(d_{1}, \ldots, d_{n}\right)$. As before, we set $n=2 m+1$.

(i) $d_{1}=\cdots=d_{n-3}=1$ and $d_{n-2}=d_{n-1}=d_{n}=0$.

(ii) $d_{1}=2 k, d_{2}=\cdots=d_{n-2 k-2}=1$ and $d_{n-2 k-1}=\cdots=d_{n}=0$, where $1 \leq$ $k \leq m-1$ and $n=2 m+1$.

If $\left(d_{1}, \ldots, d_{n}\right)$ is of the type (i), then we write $\left\langle\tau_{d_{1}} \cdots \tau_{d_{n}}\right\rangle$ by $\left\langle\rho_{n, 0}\right\rangle$. On the other hand, if $\left(d_{1}, \ldots, d_{n}\right)$ is of the type (ii), then we write $\left\langle\tau_{d_{1}} \cdots \tau_{d_{n}}\right\rangle$ by $\left\langle\rho_{n, 2 k}\right\rangle$. Thus:

$$
\left\{\begin{array}{l}
\left\langle\rho_{n, 0}\right\rangle=\left\langle z_{1} \cdots z_{n-3}, \mu_{\mathscr{M}_{n}}\right\rangle \\
\left\langle\rho_{n, 2 k}\right\rangle=\left\langle z_{1}^{2 k} z_{2} \cdots z_{n-2 k-2}, \mu_{\mathscr{M}_{n}}\right\rangle \quad(1 \leq k \leq m-1) .
\end{array}\right.
$$

For a sequence $\left(d_{1}, \ldots, d_{n}\right)$ of nonnegative integers with $\sum_{l=1}^{n} d_{i}=n-3$, we set $d_{i}=2 \alpha_{i}+\varepsilon_{i}(1 \leq i \leq n)$, where $\varepsilon_{i}=0$ or 1 . Then we have the following:

THEOREM 2.5 [7]. We have the following relations in $H^{*}\left(\mathscr{M}_{n} ; \boldsymbol{R}\right)$.

(i) If $\alpha_{i}=0$ for $1 \leq i \leq n$, then we have

$$
\left\langle\tau_{d_{1}} \cdots \tau_{d_{n}}\right\rangle=\left\langle\rho_{n, 0}\right\rangle .
$$

(ii) If $\alpha_{i} \neq 0$ for some $i$, then we have

$$
\left\langle\tau_{d_{1}} \cdots \tau_{d_{n}}\right\rangle=\left\langle\rho_{n, 2\left(\alpha_{1}+\cdots+\alpha_{n}\right)}\right\rangle .
$$

Thus it suffices to determine $\left\langle\rho_{n, 2 k}\right\rangle(0 \leq k \leq m-1)$ in order to determine the intersection numbers. About this, we have the following theorem.

THEOREM 2.6 [7]. When $n=2 m+1$, the number $\left\langle\rho_{n, 2 k}\right\rangle(0 \leq k \leq m-1)$ is given as follows.

$$
\left\langle\rho_{n, 2 k}\right\rangle=(-1)^{k} \frac{\left(\begin{array}{c}
m-1 \\
k
\end{array}\right)\left(\begin{array}{c}
2 m-1 \\
m
\end{array}\right)}{\left(\begin{array}{c}
2 m-1 \\
2 k+1
\end{array}\right)} .
$$


Example 2.7. We have the following examples:

(i) $\mathscr{M}_{5}:\left\langle\rho_{5,0}\right\rangle=1$ and $\left\langle\rho_{5,2}\right\rangle=-3$.

(ii) $\mathscr{M}_{7}:\left\langle\rho_{7,0}\right\rangle=2,\left\langle\rho_{7,2}\right\rangle=-2$ and $\left\langle\rho_{7,4}\right\rangle=10$.

(iii) $\mathscr{M}_{9}:\left\langle\rho_{9,0}\right\rangle=5,\left\langle\rho_{9,2}\right\rangle=-3,\left\langle\rho_{9,4}\right\rangle=5$ and $\left\langle\rho_{9,6}\right\rangle=-35$.

Finally we give $c\left(\mathscr{M}_{n}\right)$, the total Chern class of the tangent bundle of $\mathscr{M}_{n}$.

THEOREM 2.8 [2]. We have

$$
c\left(\mathscr{M}_{n}\right)=\left(1-z_{1}^{2}\right)^{-1} \prod_{l=1}^{n}\left(1+z_{l}\right) .
$$

Note that we have $z_{1}^{2}=\cdots=z_{n}^{2}$ (cf. Theorem 2.2 (i)). Hence we can replace $\left(1-z_{1}^{2}\right)^{-1}$ in Theorem 2.8 by $\left(1-z_{j}^{2}\right)^{-1}$ for any $j$ with $2 \leq j \leq n$.

Proof of Theorem 2.8. This theorem is essentially [2, p. 307]. But in [2], the result is stated in terms of other generators $R, V_{l}(1 \leq i \leq n-1) \in H^{2}\left(\mathscr{M}_{n} ; \boldsymbol{R}\right)$. So we summarize how to deduce Theorem 2.8 from [2].

In [2, p. 296], two dimensional cohomology classes $R, V_{l}(1 \leq i \leq n-1)$, which are the generators of $H^{*}\left(\mathscr{M}_{n} ; \boldsymbol{R}\right)$, are defined. Then in [2, Proposition 7.3], it is shown that

$$
z_{l}= \begin{cases}R+2 V_{l} & 1 \leq i \leq n-1 \\ -R & i=n .\end{cases}
$$

(Note that [2] uses the symbol $c_{l}$ to denote $z_{l}$ in this paper.)

Finally in [2, p. 307], the following result is proved.

$$
c\left(\mathscr{M}_{n}\right)=(1+R)^{-1} \prod_{l=1}^{n-1}\left(1+V_{l}+R\right) \prod_{J=1}^{n-1}\left(1+V_{\jmath}\right) .
$$

Then using (2.9) and the fact $z_{1}^{2}=\cdots=z_{n}^{2}$ (cf. Theorem 2.2), we see that (2.10) is equivalent to Theorem 2.8 .

Let $\sigma_{i}\left(z_{1}, \ldots, z_{n}\right) \in H^{2 i}\left(\mathscr{M}_{n} ; \boldsymbol{R}\right)$ be the elementary symmetric polynomial on $z_{1}, \ldots, z_{n} \in H^{2}\left(\mathscr{M}_{n} ; \boldsymbol{R}\right)$. Recall that $z_{1}^{2}=\cdots=z_{n}^{2}$ (cf. Theorem 2.2 (i)). We define $D^{2} \in H^{4}\left(\mathscr{M}_{n} ; \boldsymbol{R}\right)$ by

$$
z_{1}^{2}=\cdots=z_{n}^{2}=D^{2} .
$$

(Note that we shall not define $D \in H^{2}\left(\mathscr{M}_{n} ; \boldsymbol{R}\right)$, but just define $D^{2}$.) Then Theorem 2.8 implies the following:

THEOREM 2.12. For $0 \leq k \leq n-3$, we have

$$
c_{k}\left(\mathscr{M}_{n}\right)=\sum_{i=0}^{[k / 2]} D^{2 i} \sigma_{k-2 i}\left(z_{1}, \ldots, z_{n}\right)
$$


Remark 2.13. From Theorem 2.8, we see that $c_{1}\left(\mathscr{M}_{n}\right)=z_{1}+\cdots+z_{n}$. On the other hand, if we write the symplectic form on $\mathscr{M}_{n}$ by $\omega_{n}$, then a theorem of [2] tells us that $\left[\omega_{n}\right]=z_{1}+\cdots+z_{n} \in H^{2}\left(\mathscr{M}_{n} ; \boldsymbol{R}\right)$. Hence we have $c_{1}\left(\mathscr{M}_{n}\right)=\left[\omega_{n}\right]$.

\section{Proof of Theorem B}

For $n=5,7$ and 9 , the Chern numbers of $\mathscr{M}_{n}$ are given by the following:

THEOREM 3.1. (i) $c_{1}^{2}\left[\mathscr{M}_{5}\right]=5$ and $c_{2}\left[\mathscr{M}_{5}\right]=7$.

(ii) For $\mathscr{M}_{7}$, we have the following table of the Chern numbers.

\begin{tabular}{|c|c|}
\hline & Chern number \\
\hline$c_{1}^{4}\left[\mathscr{M}_{7}\right]$ & 154 \\
\hline$c_{1}^{2} c_{2}\left[\mathscr{M}_{7}\right]$ & 112 \\
\hline$c_{1} c_{3}\left[\mathscr{M}_{7}\right]$ & 56 \\
\hline$c_{2}^{2}\left[\mathscr{M}_{7}\right]$ & 136 \\
\hline$c_{4}\left[\mathscr{M}_{7}\right]$ & 38 \\
\hline
\end{tabular}

(iii) For $\mathscr{M}_{9}$, we have the following table of the Chern numbers.

\begin{tabular}{|c|c|}
\hline & Chern number \\
\hline$c_{1}^{6}\left[\mathscr{M}_{9}\right]$ & 13005 \\
\hline$c_{1}^{4} c_{2}\left[\mathscr{M}_{9}\right]$ & 7857 \\
\hline$c_{1}^{3} c_{3}\left[\mathscr{M}_{9}\right]$ & 3393 \\
\hline$c_{1}^{2} c_{2}^{2}\left[\mathscr{M}_{9}\right]$ & 5157 \\
\hline$c_{1}^{2} c_{4}\left[\mathscr{M}_{9}\right]$ & 1287 \\
\hline$c_{1} c_{2} c_{3}\left[\mathscr{M}_{9}\right]$ & 2421 \\
\hline$c_{1} c_{5}\left[\mathscr{M}_{9}\right]$ & 423 \\
\hline$c_{2}^{3}\left[\mathscr{M}_{9}\right]$ & 4969 \\
\hline$c_{2} c_{4}\left[\mathscr{M}_{9}\right]$ & 1459 \\
\hline$c_{3}^{2}\left[\mathscr{M}_{9}\right]$ & 1221 \\
\hline$c_{6}\left[\mathscr{M}_{9}\right]$ & 187 \\
\hline
\end{tabular}


Proof. This theorem follows from Theorems 2.2, 2.5, 2.6 and 2.12. As an example, we show $c_{1} c_{3}\left[\mathscr{M}_{7}\right]$. From Theorem 2.12, we have $c_{1}\left(\mathscr{M}_{7}\right)=$ $\sigma_{1}\left(z_{1}, \ldots, z_{7}\right)$ and $c_{3}\left(\mathscr{M}_{7}\right)=\sigma_{3}\left(z_{1}, \ldots, z_{7}\right)+D^{2} \sigma_{1}\left(z_{1}, \ldots, z_{7}\right)$. Hence

$$
c_{1} c_{3}\left[\mathscr{M}_{7}\right]=\left\langle\sigma_{1}\left(z_{1}, \ldots, z_{7}\right) \sigma_{3}\left(z_{1}, \ldots, z_{7}\right), \mu_{\mathscr{M}_{7}}\right\rangle+\left\langle D^{2} \sigma_{1}\left(z_{1}, \ldots, z_{7}\right)^{2}, \mu_{\mathscr{M}_{7}}\right\rangle .
$$

Using Theorem 2.5 and Example 2.7 (ii), we have

$$
\begin{aligned}
& \left\langle\sigma_{1}\left(z_{1}, \ldots, z_{7}\right) \sigma_{3}\left(z_{1}, \ldots, z_{7}\right), \mu_{\mathscr{M}_{7}}\right\rangle \\
& \quad=7\left\langle z_{1} \sigma_{3}\left(z_{1}, \ldots, z_{7}\right), \mu_{\mathscr{M}_{7}}\right\rangle \\
& \quad=7\left(\left(\begin{array}{l}
6 \\
2
\end{array}\right)\left\langle\rho_{7,2}\right\rangle+\left(\begin{array}{l}
6 \\
3
\end{array}\right)\left\langle\rho_{7,0}\right\rangle\right) \\
& \quad=70 .
\end{aligned}
$$

Similarly, we have

$$
\left\langle D^{2} \sigma_{1}\left(z_{1}, \ldots, z_{7}\right)^{2}, \mu_{\mathscr{M}_{7}}\right\rangle=-14
$$

Hence we have $c_{1} c_{3}\left[\mathscr{M}_{7}\right]=56$.

Now we complete the proof of Theorem B. It is known that $\Omega_{*}^{U}$ is the integral polynomial ring on classes $x_{l}$ of dimension $2 i$ for each integer $i . \Omega_{*}^{U}$ is determined by the Chern numbers. Moreover, $\Omega_{*}^{U} \otimes \boldsymbol{Q}$ is the rational polynomial ring on the cobordism classes of complex projective spaces [14].

We consider $\mathscr{M}_{7}$. The above fact tells us that in $\Omega_{8}^{U} \otimes Q,\left[\mathscr{M}_{7}\right]$ is a linear combination of $\left[\left(\boldsymbol{C} P^{1}\right)^{4}\right],\left[\left(\boldsymbol{C} P^{1}\right)^{2} \times C P^{2}\right],\left[\boldsymbol{C} P^{1} \times \boldsymbol{C} P^{3}\right],\left[\left(\boldsymbol{C} P^{2}\right)^{2}\right]$ and $\left[\boldsymbol{C} P^{4}\right]$. The coefficients are determined completely since we know all Chern numbers of $\mathscr{M}_{7}$ in Theorem 3.1 (ii). Thus we get a description of $\left[\mathscr{M}_{7}\right]$ in $\Omega_{8}^{U} \otimes Q$, and the result is given in Theorem $\mathrm{B}$ (ii). Since the coefficients of the description are integers, this is also a description in $\Omega_{8}^{U}$.

Similarly, we can prove Theorem B (i) and (iii).

Remark 3.2. In order to get a description of $\mathscr{M}_{9}$ in $\Omega_{12}^{U}$, we define an element $x_{5}$ of $\Omega_{10}^{U}$ by

$$
x_{5}=\left[C P^{5}\right]+\left[H_{3,3}\right]-\left[H_{2,4}\right],
$$

where $H_{a, b}$ denotes a non-singular hypersurface of degree $(1,1)$ in $\boldsymbol{C} P^{a} \times C P^{b}$. Since $s_{5}\left(x_{5}\right)=1$, we see that $x_{5} \in \Omega_{10}^{U}$ is a ring generator. Then it is easy to see that in $\Omega_{10}^{U}$, we have

$$
\begin{aligned}
{\left[\boldsymbol{C} P^{5}\right]=} & 21\left[\left(\boldsymbol{C} P^{1}\right)^{5}\right]-68\left[\left(\boldsymbol{C} P^{1}\right)^{3} \times C P^{2}\right]+27\left[\left(\boldsymbol{C} P^{1}\right)^{2} \times C P^{3}\right] \\
& +51\left[C P^{1} \times\left(C P^{2}\right)^{2}\right]-6\left[C P^{1} \times C P^{4}\right]-30\left[C P^{2} \times C P^{3}\right]+6 x_{5} .
\end{aligned}
$$

If we put (3.3) into Theorem B (iii), then we get the following description of $\left[\mathscr{M}_{9}\right]$ in $\Omega_{12}^{U}$. 


$$
\begin{aligned}
{\left[\mathscr{M}_{9}\right]=} & 3123\left[\left(\boldsymbol{C} P^{1}\right)^{6}\right]-10196\left[\left(\boldsymbol{C} P^{1}\right)^{4} \times \boldsymbol{C} P^{2}\right]+4194\left[\left(\boldsymbol{C} P^{1}\right)^{3} \times \boldsymbol{C} P^{3}\right] \\
& +7700\left[\left(\boldsymbol{C} P^{1}\right)^{2} \times\left(\boldsymbol{C} P^{2}\right)^{2}\right]-1100\left[\left(\boldsymbol{C} P^{1}\right)^{2} \times \boldsymbol{C} P^{4}\right] \\
& -4620\left[\boldsymbol{C} P^{1} \times \boldsymbol{C} P^{2} \times \boldsymbol{C} P^{3}\right]+0\left[\left(\boldsymbol{C} P^{2}\right)^{3}\right]+0\left[\boldsymbol{C} P^{2} \times \boldsymbol{C} P^{4}\right] \\
& +55\left[\left(\boldsymbol{C} P^{3}\right)^{2}\right]-35\left[\boldsymbol{C} P^{6}\right]+880\left[\boldsymbol{C} P^{1}\right] \cdot x_{5} .
\end{aligned}
$$

\section{Proof of Theorem $\mathrm{C}$}

Proof of Theorem $C$ (i). From Remark 2.13, we have $c_{1}\left(\mathscr{M}_{n}\right)=\left[\omega_{n}\right]$. In [7], the symplectic volume $\left\langle\omega_{n}^{n-3}, \mu_{\mathscr{M}_{n}}\right\rangle$ is determined. Hence Theorem C (i) follows.

Proof of Theorem $C$ (ii). Note that $c_{n-3}\left[\mathscr{M}_{n}\right]=\chi\left(\mathscr{M}_{n}\right)$, the Euler characteristic of $\mathscr{M}_{n}$. For odd $n, H_{*}\left(\mathscr{M}_{n} ; \boldsymbol{R}\right)$ is determined in [10], [12]. Hence Theorem C (ii) follows.

Proof of Theorem $C$ (iii). We can deduce this theorem from direct calculations using Theorems $2.5,2.6$ and 2.8 , or from the fact $h^{p, q}\left(\mathscr{M}_{n}\right)=0$ for $p \neq q[10],[12]$.

We can also deduce this theorem from the following stronger assertion. (Recall that the Todd genus is birational invariant [3].)

ASSERTION 4.1. $\mathscr{M}_{n}$ is birationally equivalent to $C P^{n-3}$.

Proof. In order to construct a birational map $f: \mathscr{M}_{n} \rightarrow C P^{n-3}$, it is convenient to substitute $\mathscr{M}_{n}$ by a space $\mathscr{N}_{n}$, which is biholomorphic to $\mathscr{M}_{n}$. Recall that an element $P=\left(x_{1}, \ldots, x_{n}\right)$ of $\left(C P^{1}\right)^{n}$ is semistable if and only if $P$ contains no point of $C P^{1}$ with multiplicity strictly greater than $n / 2$. Let $\mathscr{N}_{n}$ be the orbit space of semistable points in $\left(C P^{1}\right)^{n}$ with respect to the natural action of the group $\operatorname{PSL}(2, C)$. Thus:

$$
\mathscr{N}_{n}=\left\{P=\left(x_{1}, \ldots, x_{n}\right) \in\left(C P^{1}\right)^{n}: P \text { is semistable }\right\} / P S L(2, C) .
$$

Then it is known that $\mathscr{M}_{n}$ is biholomorphic to $\mathscr{N}_{n}$ [8], [10], [12]:

$$
\mathscr{M}_{n} \cong \mathscr{N}_{n}
$$

Now we construct a rational map $\phi: \mathscr{N}_{n} \rightarrow C P^{n-3}$ in the same way as in [9, p. 134]. (The inverse rational map $C P^{n-3} \rightarrow \mathscr{N}_{n}$ is constructed similarly.) Let $P=\left(x_{1}, \ldots, x_{n}\right) \in \mathscr{N}_{n}$. By the $\operatorname{PSL}(2, C)$-action, we can assume that $x_{1}=\infty$. Thus:

$$
\mathscr{N}_{n}=\left\{P=\left(x_{1}, \ldots, x_{n}\right) \in\left(C P^{1}\right)^{n}: P \text { is semistable and } x_{1}=\infty\right\} / G,
$$

where $G$ is a subgroup of $\operatorname{PSL}(2, C)$ defined by 


$$
G=\left\{\left(\begin{array}{cc}
z & 0 \\
\xi & z^{-1}
\end{array}\right): z \in C^{*}, \xi \in C\right\} .
$$

Let $\mathscr{N}_{n}^{\prime}$ be the subspace of $\mathscr{N}_{n}$ defined by

$$
\begin{aligned}
\mathscr{N}_{n}^{\prime}= & \left\{P=\left(x_{1}, \ldots, x_{n}\right) \in\left(C P^{1}\right)^{n}: P \text { is semistable and } x_{1}=\infty,\right. \\
& \left.x_{l} \neq \infty(2 \leq i \leq n)\right\} / G .
\end{aligned}
$$

Then we define a map $\phi: \mathscr{N}_{n}^{\prime} \rightarrow C P^{n-3}$ as follows. Let $P=\left(x_{1}, \ldots, x_{n}\right) \in \mathscr{N}_{n}^{\prime}$. Note that $\boldsymbol{C} P^{1}-\{\infty\}$ is isomorphic to $\boldsymbol{C}$. Since $x_{l} \neq \infty(2 \leq i \leq n)$, we can regard that $x_{l} \in C(2 \leq i \leq n)$. There is exactly one point $z=z\left(x_{1}, \ldots, x_{n}\right) \in$ $C P^{1}-\{\infty\}=C$ such that $\sum_{l=2}^{n}\left(z-x_{l}\right)=0$. Then the point $\phi\left(x_{1}, \ldots, x_{n}\right)$ is defined to be point with homogeneous coordinates $\left(z-x_{2}, \ldots, z-x_{n}\right)$. This completes the proof of Assertion 4.1.

Proof of Theorem $C$ (iv). Recall that $s_{n-3}\left[\mathscr{M}_{n}\right]$ is a characteristic number defined from the Chern classes. Let $n=2 m+1$ and let $s_{m-1}(p)\left[\mathscr{M}_{n}\right]$ be the characteristic number which is defined in the same way as in $s_{n-3}\left[\mathscr{M}_{n}\right]$ but using the Pontrjagin classes instead of the Chern classes. Then it is known that $s_{n-3}\left[\mathscr{M}_{n}\right]=s_{m-1}(p)\left[\mathscr{M}_{n}\right]$ (see for example [13]).

Since $\mathscr{M}_{n}$ is oriented cobordant to $(-1)^{m+1}\left(\begin{array}{c}2 m-1 \\ m\end{array}\right) C P^{2 m-2} \quad[6]$ and $s_{m-1}(p)\left[C P^{2 m-2}\right]=2 m-1$, Theorem $\mathrm{C}$ (iv) follows.

Finally we give some more results on the Chern numbers.

THEOREM 4.5. We have the following formulae for $n=2 m+1$.

(i) We have

$$
c_{1} c_{n-4}\left[\mathscr{M}_{n}\right]=-(2 m+1) 2^{2 m-1}+(2 m+1)(m+1)\left(\begin{array}{c}
2 m-1 \\
m
\end{array}\right) .
$$

(ii) We have

$$
c_{2} c_{n-5}\left[\mathscr{M}_{n}\right]=-\left(2 m^{2}+m+1\right) 2^{2 m-1}+\frac{(2 m+1)\left(3 m^{2}+2 m+3\right)}{3}\left(\begin{array}{c}
2 m-1 \\
m
\end{array}\right) \text {. }
$$

Recall Theorem C (ii). In general, it is easy to see that $c_{i} c_{n-3-i}\left[\mathscr{M}_{n}\right]$ $(i \leq(n-3) / 2)$ is of the form

$$
c_{i} c_{n-3-i}\left[\mathscr{M}_{n}\right]=-f_{i}(m) 2^{2 m-1}+(2 m+1) g_{i}(m)\left(\begin{array}{c}
2 m-1 \\
m
\end{array}\right),
$$

where $f_{i}(m)$ and $g_{i}(m)$ are polynomials of degree $i$ with variable $m$.

We can prove Theorem 4.5 in the same way as in the proof of Theorem 3.1 using Theorems 2.5, 2.6 and 2.12 . 


\section{REFERENCES}

[1] M. BRion, Cohomologie équivariante des points semı-stables, J. Reıne Angew. Math., 421 (1991), 125-140.

[2] J-C. Hausmann and A. KnUtson, The cohomology ring of polygon spaces, Ann. Inst. Fourier (Grenoble), 48 (1998), 281-321.

[ 3 ] F Hirzebruch, Topological Methods in Algebraic Geometry 3rd ed., translated, Springer, Berlin, 1966.

[4] Y KamiYama, Topology of equilateral polygon linkages, Topology Appl., 68 (1996), 13-31.

[5] Y. KamiYama, The homology of singular polygon spaces, Canad. J. Math., 50 (1998), 581594.

[6] Y Kamiyama, Remarks on the topology of spatial polygon spaces, Bull. Austral. Math. Soc., 58 (1998), 373-382.

[7] Y. KamiYama AND M. TezuKa, Symplectic volume of the moduli space of spatial polygons, J. Math. Kyoto Univ., 39 (1999), 557-575.

[ 8 ] M. Kapovich AND J. Millson, The symplectic geometry of polygons in Euclidean space, J. Differential Geom., 44 (1996), 479-513.

[9] M. KaPranov, The permutoassociahedron, MacLane's coherence theorem and asymptotic zones for the $K Z$ equation, J. Pure Appl. Algebra, 85 (1993), 119-142.

[10] F KIRWAN, Cohomology of Quotients in Symplectic and Algebraic Geometry, Mathematical Notes 31, Princeton University Press, Princeton, 1984.

[11] F KIRWAN, The cohomology rings of moduli spaces of bundles over Riemann surfaces, $\mathbf{J}$. Amer. Math. Soc., 5 (1992), 853-906.

[12] A. KlyachKo, Spatial polygons and stable configurations of points in the projective line, Algebraic Geometry and its Applications Yaroslavl' 1992 (A. Tikhomirov and A. Tyurın eds.), Aspects Math. E 25, Vieweg, Braunschwe1g, 1994, pp. 67-84.

[13] J. Milnor and J. Stasheff, Characterıstic Classes, Princeton University Press, Princeton, 1974.

[14] R. Stong, Notes on Cobordism Theory, Princeton University Press, Princeton, 1968.

DEPARTMENT OF MATHEMATICS

UNIVERSITY OF THE RYUKYUS

NishiHaRa-CHO, OKINAWA

903-01, JAPAN

E-mail: kamıyama@sci.u-ryukyu.ac.jp 